\title{
Long-Term Benefit of Mesalamine Granules for Patients Who Achieved Corticosteroid-Induced Ulcerative Colitis Remission
}

\author{
Gary R. Lichtenstein ${ }^{1}$ - Glenn L. Gordon ${ }^{2} \cdot$ Salam Zakko $^{3}$ - Uma Murthy ${ }^{4}$. \\ Shahriar Sedghi ${ }^{5} \cdot$ Ron Pruitt $^{6} \cdot$ Andrew C. Barrett $^{7} \cdot$ Enoch Bortey $^{7}$. \\ Craig Paterson $^{7} \cdot$ William P. Forbes ${ }^{7}$
}

Received: 11 April 2015/Accepted: 3 September 2015/Published online: 12 November 2015

(C) The Author(s) 2015. This article is published with open access at Springerlink.com

\begin{abstract}
Background Patients with ulcerative colitis (UC) who achieve remission with corticosteroids often relapse after tapering or discontinuation; alternative treatments limiting steroid exposure and UC relapse would be beneficial. It remains uncertain whether patients with corticosteroid-induced remission experience benefit with mesalamine granules (MG), a locally acting aminosalicylate extendedrelease capsule formulation for maintenance of UC remission in adults.

Aims Efficacy and safety of MG $1.5 \mathrm{~g}$ once daily was evaluated in patients with UC in corticosteroid-induced remission.

Methods Data from patients with previous corticosteroid use to achieve baseline UC remission were analyzed from two 6-month randomized, double-blind, placebo-controlled trials and a 24-month open-label extension (OLE). Six-
\end{abstract}

Gary R. Lichtenstein

grl@uphs.upenn.edu

1 Division of Gastroenterology, Hospital of the University of Pennsylvania, University of Pennsylvania School of Medicine, 7th Floor Perelman Center, Room 753, 3400 Civic Center Boulevard, Philadelphia, PA 19104, USA

2 Center for Digestive and Liver Diseases, Mexico, MO, USA

3 Connecticut Gastroenterology Institute and Clinical Research Foundation, Bristol, CT, USA

4 Syracuse VA Medical Center, Syracuse, NY, USA

5 Gastroenterology Associates of Central Georgia, LLC, Macon, GA, USA

6 Nashville Medical Research Institute, The Maria Nathanson Center at Saint Thomas Hospital, Nashville, TN, USA

7 Salix, a Division of Valeant Pharmaceuticals North America LLC, Bridgewater, NJ, USA month relapse-free rates were assessed using the revised Sutherland Disease Activity Index. UC-related adverse events (AEs) were recorded during the 30 months.

Results Included were 158 steroid-treated patients in UC remission (MG, $n=105$; placebo, $n=53$ ) and 74/105 MG-treated patients who continued MG in the OLE. A significantly larger percentage of patients remained relapse-free at 6 months with MG $(77.1 \%)$ versus placebo $(54.7 \% ; P=0.006)$, with a $55 \%$ reduction in relapse risk (hazard ratio [HR] $0.45 ; 95 \%$ CI $0.25-0.79$ ). There was a similar $(49.2 \%)$ reduction in risk of UC-related AEs at 6 months (HR $0.51 ; 95 \%$ CI $0.31-0.84 ; P=0.009$ ) that was sustained during the OLE.

Conclusions MG $1.5 \mathrm{~g}$ once daily administered for maintenance of corticosteroid-induced remission was associated with low risk of relapse and UC-related AEs. ClinicalTrials.gov NCT00744016, NCT00767728, and NCT00326209.

Keywords Inflammatory bowel diseases - Mesalamine . Remission · Steroids · Ulcerative colitis

\section{Introduction}

Ulcerative colitis (UC) is a chronic, progressive gastrointestinal (GI) disease characterized by diffuse, uncontrolled mucosal inflammation [1-3]. Its clinical course is characterized by unpredictable periods of disease flare, and remission [2]. The typical UC flare presents as a constellation of symptoms, including rectal urgency, bloody diarrhea, abdominal cramps, tenesmus, fatigue, and weight loss [1-3]. The estimated incidence of UC in North America ranges from 2.3 to 15.6 cases per 100,000 patientyears, and the prevalence rate is $37.5-246$ cases per 
100,000 individuals [4]. Notably, UC is associated with substantial morbidity, disability, and economic burden, with estimated annual costs approaching $\$ 2.7$ billion in the USA (measured in 2003-2004 US dollars) [2, 5, 6].

Oral 5-aminosalicylic acid (5-ASA) formulations are considered first-line options for the induction and maintenance of remission of mild-to-moderate UC [2]. Several formulations have been developed to help improve patient adherence (e.g., a formulation with reduced dosing frequency) and to more directly target a higher concentration of active agent to the colon. Mesalamine granules (MG), an extended-release capsule formulation (Apriso ${ }^{\circledR}$; Salix Pharmaceuticals, Inc., Raleigh, NC), is indicated for the maintenance of UC remission in adults [7]. This formulation combines a delayed- and extended-release delivery mechanism [7, 8]. Specifically, each granule is designed to initiate the release of mesalamine at a $\mathrm{pH} \geq 6$, and the polymer matrix facilitates a slow, sustained release of mesalamine in the terminal ileum and throughout the colon. [8].

Two identically designed, double-blind, placebo-controlled studies have demonstrated the efficacy of once-daily MG (1.5 g/day) for the maintenance of UC remission, with significantly more patients treated with $\mathrm{MG}$ remaining relapse-free at 6 months compared with placebo in the pooled analysis (79.4 vs. $62.4 \%$, respectively; $P<0.001)$ $[9,10]$.

Systemic corticosteroids may be administered for the induction of remission in cases of acute relapse of UC, especially when an oral 5-ASA is ineffective, or for patients presenting with severe symptoms [2]. However, the adverse effect profile of corticosteroids has led healthcare providers to limit their use when possible and to seek safer long-term alternatives. Furthermore, patients with UC who achieve remission with corticosteroid treatment often experience a relapse of symptoms during tapering or soon after discontinuation [11]. Therefore, a steroid-sparing therapy for the treatment of UC might aid in reducing the burden of UC.

The purpose of this analysis was to evaluate the shortand long-term efficacy and safety of once-daily MG in patients who had previously received corticosteroid therapy to achieve their UC remission. Patients in this analysis participated in two randomized, double-blind, placebocontrolled Phase 3 trials [9], including a cohort of these patients who enrolled in a 24-month open-label extension (OLE) trial [12].

\section{Methods}

Data were analyzed from three multicenter trials, including two identically designed, randomized, double-blind, 6-month, placebo-controlled Phase 3 trials (MPUC3003 and MPUC3004) and a 24-month OLE trial (MPUC3005), all of which were registered under ClinicalTrials.gov identifiers NCT00744016, NCT00767728, and NCT00326209, respectively [9, 12, 13]. Patient populations and study design for the three trials have been previously published and are briefly described in this manuscript [9, 12]. All patients included in the current analysis had a history of previous steroid use to achieve their current UC remission and, upon enrollment, satisfied the entry criteria for all studies as summarized below. Patients enrolled in the OLE trial who were included in this pooled analysis had been previously enrolled in one of the double-blind trials.

\section{Patients and Treatment}

Male and non-pregnant, non-lactating female patients $\geq 18$ years of age were eligible for participation in the double-blind trials if they had a confirmed diagnosis of mild-to-moderate UC, determined using the revised Sutherland Disease Activity Index (SDAI) [7, 14], with defined subscale scores at screening (rectal bleeding score of 0 and mucosal appearance score of 0 or 1 ). In addition, all patients had a history of at least 1 flare requiring therapeutic intervention within the past 1-12 months and were documented to be in remission for $>1$ month but $<12$ months. Primary exclusion criteria included receipt of chronic immunosuppressive therapy or corticosteroids (oral, rectal, intravenous) within 30 days prior to screening.

Forty-eight centers participated in trial MPUC3003, 40 in MPUC3004, and 66 in MPUC3005. The double-blind trials started in December 2004 and were completed by April or August 2007; the OLE trial started in December 2005 and was completed in May 2008. Each protocol was approved by institutional review boards or ethics committees and conducted in accordance with International Conference on Harmonisation guidelines and other applicable laws and regulations. All patients provided written informed consent.

In the double-blind trials, patients were randomly assigned to consecutive treatment numbers allocated in the order of enrollment and in a 2:1 ratio received MG $1.5 \mathrm{~g}$ (dosed as four capsules, each containing $0.375 \mathrm{~g}$ of mesalamine) or matching placebo once daily. The use of concomitant medications including immunosuppressants, chronic non-steroidal anti-inflammatory drugs, corticosteroids, oral antibiotics (except as 7- to 10-day courses for conditions unrelated to UC), psyllium-containing compounds, and other 5-ASA formulations was prohibited in the double-blind trials. The investigators, patients, and research staff members (including project biostatisticians) were blinded to study medication assignment until after database lock at the end of each study. The OLE was unblinded, and all patients received MG $1.5 \mathrm{~g}$ once daily. 


\section{Assessments}

The double-blind trials consisted of a 1-week screening phase, a 6-month treatment phase, and a follow-up visit ( 2 weeks after the end-of-treatment phase). The OLE trial consisted of a 24-month treatment phase and a follow-up visit 2 weeks after the last dose of MG. The 6-month treatment phase of the double-blind, randomized trials consisted of four clinic visits to assess disease activity and monitor adverse events (AEs) at baseline and months 1, 3, and 6. The 24-month OLE trial consisted of clinic visits to monitor AEs at baseline, months 1, 3, and 6, and then every 3 months for up to 24 months. In addition, during the OLE trial, telephone interviews were conducted at week 2, months 2, 4, and 5, and then at every subsequent month between clinic visits.

For the double-blind trials, a complete UC disease activity assessment, including flexible sigmoidoscopy, was performed at screening or baseline and at the 6-month visit using the revised SDAI (all 4 components) [7, 14]. The revision to the SDAI was the deletion of "friability" from a mucosal appearance score equal to 1 to clarify the definition of remission. The revised SDAI total score evaluated each of the following four parameters on a scale of $0-3$, with a maximum total score of 12: (1) stool frequency (i.e., 0 to $>4$ stools/day more than normal); (2) rectal bleeding (i.e., none to mostly blood); (3) mucosal appearance (i.e., intact mucosa with preserved or distorted vessels to blood in lumen, gross ulceration, exudates), and (4) physician's rating of disease activity (i.e., normal to severe).

In the OLE trial, only patients entering the trial more than 30 days after having completed the double-blind trial were screened using the revised SDAI to confirm remission; for the remainder of patients, the baseline SDAI from the end-of-treatment visit for the double-blind trial was used. AEs were monitored throughout the three trials.

\section{Endpoints and Data Analyses}

Three populations of patients with a history of previous corticosteroid use were analyzed: (1) the pooled intent-totreat (ITT) population, defined as randomized patients in either of the double-blind trials who received at least one dose of study medication and had a revised SDAI response at the 6-month endpoint visit; (2) the short-term safety population, defined as the ITT population with at least one post-baseline safety assessment, and (3) the long-term safety population, defined as patients who completed one of the double-blind trials, received at least one dose of MG during the OLE trial and had at least one post-baseline safety assessment.

Demographics and baseline disease characteristics, exposure, and safety data were summarized using descriptive statistics. The primary efficacy endpoint during the double-blind trials was the percentage of patients who remained relapse-free from baseline through the 6-month evaluation. Relapse was defined as a revised SDAI individual component score for rectal bleeding of $\geq 1$ and mucosal appearance score $\geq 2$, or early study termination when the reason for termination was lack of efficacy or discontinuation due to a UC-related AE. The treatment groups were compared for the primary endpoint using a Cochran-Mantel-Haenszel test.

Secondary efficacy endpoints included mean change from baseline in the revised total SDAI score at the 6-month endpoint; change from baseline at month 6 in the subcomponents of the SDAI score (rectal bleeding score, mucosal appearance score, physician's rating of disease activity, and stool frequency); percentage of patients classified as achieving treatment success (defined as maintaining an SDAI total score $\leq 2$ with no individual component $>1$ and a rectal bleeding score of 0 at month 6); and the 6-month cumulative relapse-free probability.

Statistical analyses of the secondary endpoints were performed in a predefined hierarchical fashion until a nonsignificant $P$ value was identified $(P>0.05)$, after which point significance tests were considered exploratory. For relapse-free duration, a Cox proportional hazards regression model was used to assess differences between treatment groups, a hazard ratio (HR) with associated $95 \% \mathrm{CI}$ was generated to determine reduction in risk (1-HR) over the first 6 months of treatment, and Kaplan-Meier methods were used to calculate cumulative relapse-free probability estimates.

Although no formal efficacy assessments were performed in the OLE trial, the effects of MG on maintenance of UC remission were assessed by evaluating UC-related AEs, which were defined as UC, hematochezia, and frequent bowel movements. The percentage of patients in the short- and long-term safety populations who remained free from UC-related AEs for the double-blind and OLE trials was assessed from start of MG therapy through 6 and 30 months of therapy. UC-related, AE-free duration was computed as the number of days between the start of MG or placebo use (double-blind studies only), and the date on which at least 1 of the predefined UC-related AEs was first detected, or the date of early termination from the trial, plus 1 day. Patients who completed 6 and 30 months of treatment, or who withdrew from the trial without evidence of a UC-related AE, were censored at the time of their final evaluation; censoring time was calculated as the date of final evaluation minus the start of study drug, plus 1 day.

For the short-term safety population, a Cox proportional hazards regression model was used to assess differences in UC-related, AE-free duration between MG and placebo. The HR and associated $95 \%$ CI were obtained from this model to 
determine reduction in risk (1-HR) over the first 6 months of treatment. Cumulative UC-related, AE-free probability estimates were calculated using Kaplan-Meier methods.

Person-years of exposure (PYE) was calculated as ([mean exposure in days $\div 365.25] \times$ no. of patients). PYE rate was calculated as (events $\div$ PYE). Mean percent compliance was calculated using the following equation: patients) were evaluated for UC-related AE events during 6 months of treatment (short-term safety population); four patients were excluded because post-baseline safety data were not available. Seventy-four of the 102 (72.5\%) MGtreated patients entered the OLE trial and were evaluated for UC-related AEs during an additional 24 months of therapy (months 6-30; long-term safety population).

$\%$ compliance $=\frac{(\text { no. of pills dispensed }- \text { no. of pills returned })}{(4 \times \text { no. of days of exposure })} \times 100$

\section{Results}

\section{Patients}

A total of 158 of 562 patients (105 in MG group and 53 in placebo group) from the two double-blind trials reported a history of receiving corticosteroid therapy to induce UC remission $\geq 30$ days prior to enrollment and were included in the ITT population and evaluation of the primary and secondary efficacy endpoints (Fig. 1). Of these 158 patients, 154 (102 MG-treated and 52 placebo-treated

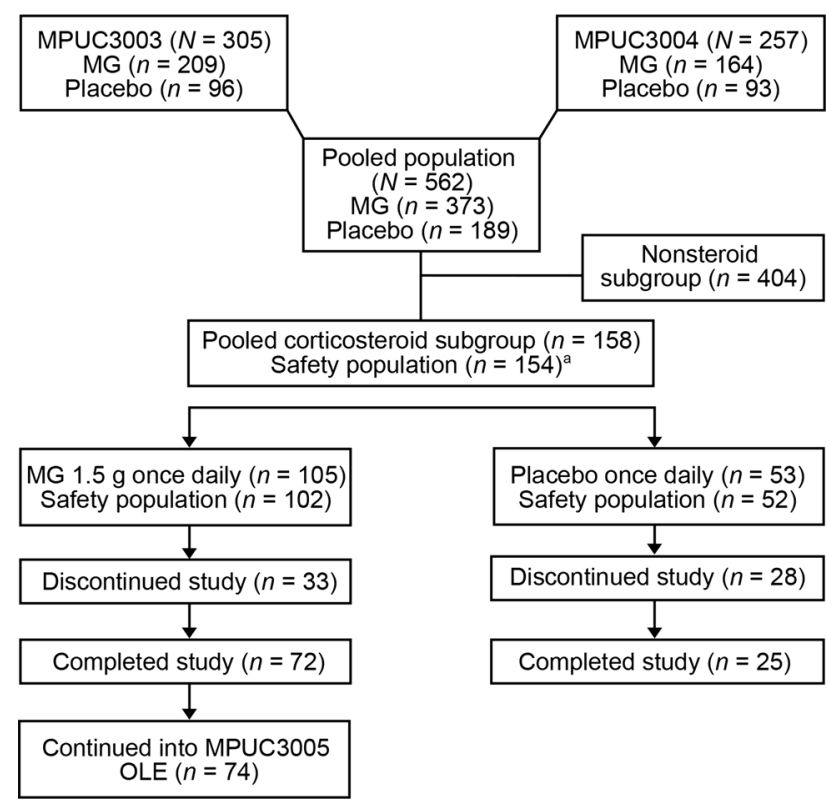

Fig. 1 Patient disposition for subpopulation of patients previously treated with corticosteroids for induction or maintenance of remission of ulcerative colitis during the two double-blind Phase 3 trials (MPUC3003 and MPUC3004) and the open-label extension trial (MPUC3005). $M G$ mesalamine granules, OLE open-label extension.

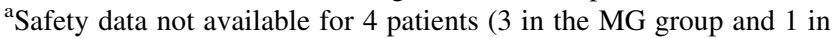
the placebo group)
Demographic and baseline disease characteristics were similar between the pooled ITT and short- and long-term safety populations (Table 1). Overall, the three cohorts of patients were predominantly white $(>92 \%)$ and in their mid-40s (45-48 years), with a slight female predominance. The mean duration of current UC remission was approximately 17-18 weeks, and mean baseline total SDAI scores were approximately $0.7-0.9$.

Adherence to once-daily MG was high among the safety populations analyzed, with mean adherence of approximately $96 \%$ during the 6-month double-blind and OLE trials. Mean exposure to MG was 436 and 623 days in the short- and long-term safety populations, respectively, and the mean total cumulative MG dose was 630 and $900 \mathrm{~g}$, respectively.

\section{Efficacy}

For the pooled ITT population of patients previously treated with corticosteroids, a significantly higher percentage of patients treated with MG $1.5 \mathrm{~g}$ were relapse-free at month 6 compared with placebo (77.1 vs. $54.7 \% ; P=0.006$ ). During the 6-month period, treatment with $\mathrm{MG}$ reduced the risk of relapse by $55 \%$ versus placebo in patients who were previously treated with corticosteroids (HR 0.45; $95 \%$ CI 0.25-0.79; Fig. 2). Significant improvements $(P \leq 0.025)$ favoring $\mathrm{MG}$ once daily were also observed for most secondary efficacy endpoints, including improvement in mean total SDAI score, rectal bleeding score, physician's disease activity rating score, and stool frequency score (Table 2).

\section{Adverse Events}

\section{UC-Related Adverse Events}

Among patients previously treated with corticosteroids in the short-term safety population, a greater percentage of patients receiving $\mathrm{MG}$ for 6 months (79.4\%) had not 
Table 1 Demographics and baseline characteristics of patients previously treated with corticosteroids for ulcerative colitis

\begin{tabular}{|c|c|c|c|}
\hline \multirow[t]{2}{*}{ Parameter } & \multicolumn{2}{|c|}{ ITT population $^{\mathrm{a}}(N=158)$} & \multirow{2}{*}{$\begin{array}{l}\text { Long-term safety } \\
\text { population }{ }^{\mathrm{a}}(N=74) \\
\text { MG } 1.5 \mathrm{~g} / \text { day }(n=74)\end{array}$} \\
\hline & $\begin{array}{l}\text { MG } 1.5 \mathrm{~g} / \text { day } \\
(n=105)\end{array}$ & Placebo $(n=53)$ & \\
\hline Age, year, mean (SD) & $45.3(14.1)$ & $48.3(15.4)$ & $47.9(13.3)$ \\
\hline \multicolumn{4}{|l|}{ Sex, $n(\%)$} \\
\hline Male & $48(45.7)$ & $24(45.3)$ & $32(43.2)$ \\
\hline Female & $57(54.3)$ & $29(54.7)$ & $42(56.8)$ \\
\hline \multicolumn{4}{|l|}{ Race, $n(\%)$} \\
\hline White & $97(92.4)$ & $49(92.5)$ & $71(95.9)$ \\
\hline Black & $7(6.7)$ & $3(5.7)$ & $3(4.1)$ \\
\hline Other & $2(1.9)$ & $1(1.9)$ & 0 \\
\hline UC duration, week, median (range) & $180.0(14.0-2049.0)$ & $160.0(17.0-2151.0)$ & $175.5(14.0-2049.0)$ \\
\hline Duration of current UC remission, week, mean (SD) & $17.4(11.7)$ & $17.0(11.6)$ & $18.4(12.3)$ \\
\hline \multicolumn{4}{|l|}{ Revised SDAI score, mean (SD) } \\
\hline Total & $0.8(0.9)$ & $0.9(1.0)$ & $0.7(0.8)$ \\
\hline Stool frequency & $0.1(0.4)$ & $0.1(0.4)$ & $0.1(0.4)$ \\
\hline Rectal bleeding & 0 & $0.0(0.1)$ & 0 \\
\hline Mucosal appearance & $0.5(0.5)$ & $0.6(0.5)$ & $0.5(0.5)$ \\
\hline Physician's rating of severity & $0.2(0.4)$ & $0.2(0.4)$ & $0.2(0.4)$ \\
\hline
\end{tabular}

ITT intent-to-treat, $M G$ mesalamine granules, SDAI Sutherland Disease Activity Index, $U C$ ulcerative colitis

${ }^{a}$ Data obtained at baseline for Phase 3 double-blind trials

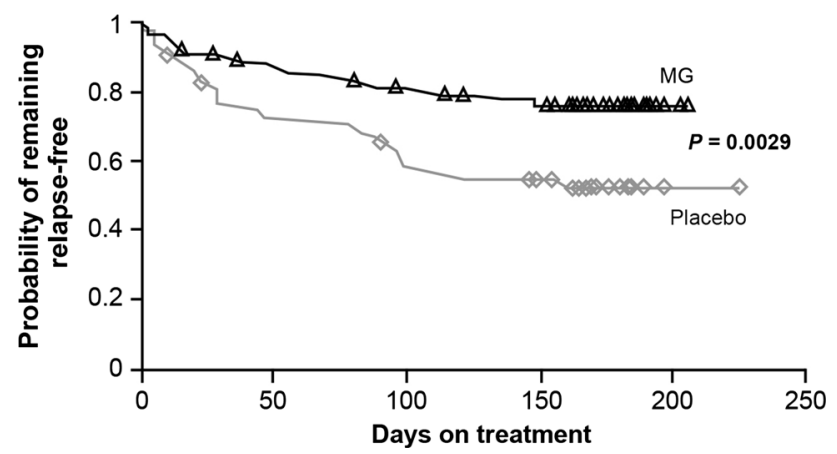

Fig. 2 Probability of remaining relapse-free during 6 months of treatment in patients previously treated with corticosteroids (MPUC3003 and MPUC3004, pooled). $P$ value determined using Poisson regression analysis. $M G$ mesalamine granules

reported UC-related AEs compared with patients receiving placebo for 6 months $(67.3 \%)$. During the 6-month period, treatment with MG resulted in a $49.2 \%$ reduction in the risk of a UC-related AE versus placebo in patients who were previously treated with corticosteroids (HR 0.51; $95 \%$ CI $0.31-0.84 ; P=0.009$ ).

For the 74 MG-treated patients who received an additional 24 months of therapy, only $12(16.2 \%)$ experienced a GI-related $\mathrm{AE}$ (14 events total), with the majority of the 12 patients $(91.7 \%)$ reporting UC. Patients in the longterm safety population treated with $\mathrm{MG}$ for up to
30 months had a statistically significantly greater probability of remaining free from UC-related AEs compared with patients treated with placebo for 6 months in the double-blind studies ( $P=0.0001$; Fig. 3$)$.

\section{Overall Adverse Events}

The incidence of any treatment-emergent AEs among patients previously treated with corticosteroids was low for patients treated for up to 6 months (short-term safety population) with MG or placebo (Table 3). When comparing the short- and long-term safety populations following MG therapy, the rate of any AE, based on PYE, was higher in patients treated with long-term compared with short-term exposure (9.6 vs. 6.0 events/PYE, respectively). However, overall drug-related $\mathrm{AE}$ rates $(0.5$ events/PYE) and rates of AEs leading to premature discontinuation from the study $(0.4$ events/PYE for patients with short-term exposure vs. 0.3 events/PYE for patients with long-term exposure) were similar between the two MG safety populations. Likewise, the rate of GI-related AEs for the MG treatment groups was comparable between patients in the short- and long-term safety populations (Table 3). Headache was the most common non-GI-related AE reported among MG patients following short- and long-term administration (rates of 0.6 and 1.0 events/PYE, respectively). Renal- and hepatic-related 
Table 2 Secondary efficacy endpoints at month $6^{a}$ for patients previously treated with corticosteroids for ulcerative colitis (ITT population)

\begin{tabular}{|c|c|c|c|}
\hline Endpoint & MG $1.5 \mathrm{~g} /$ day $(n=105)$ & Placebo $(n=53)$ & $P$ value \\
\hline Change from baseline in revised total SDAI score, mean (SD) & $0.9(2.4)$ & $2.2(3.0)$ & 0.005 \\
\hline \multicolumn{4}{|l|}{ Change from baseline in stool frequency, $n(\%)$} \\
\hline-1 & $5(4.8)$ & $1(1.9)$ & \multirow[t]{5}{*}{0.003} \\
\hline 0 & $80(76.2)$ & $30(56.6)$ & \\
\hline 1 & $10(9.5)$ & $11(20.8)$ & \\
\hline 2 & $9(8.6)$ & $6(11.3)$ & \\
\hline 3 & $1(1.0)$ & $5(9.4)$ & \\
\hline \multicolumn{4}{|l|}{ Change from baseline in rectal bleeding score, $n(\%)$} \\
\hline 0 & $85(81.0)$ & $34(64.2)$ & \multirow[t]{4}{*}{0.025} \\
\hline 1 & $12(11.4)$ & $10(18.9)$ & \\
\hline 2 & $7(6.7)$ & $8(15.1)$ & \\
\hline 3 & $1(1.0)$ & $1(1.9)$ & \\
\hline \multicolumn{4}{|l|}{ Change from baseline in mucosal appearance, $n(\%)$} \\
\hline-1 & $16(15.2)$ & $4(7.5)$ & \multirow[t]{5}{*}{0.056} \\
\hline 0 & $66(62.9)$ & $29(54.7)$ & \\
\hline 1 & $12(11.4)$ & $14(26.4)$ & \\
\hline 2 & $11(10.5)$ & $6(11.3)$ & \\
\hline 3 & 0 & 0 & \\
\hline \multicolumn{4}{|l|}{ Change from baseline in physician's rating of disease, $n(\%)$} \\
\hline-1 & $7(6.7)$ & $1(1.9)$ & \multirow[t]{5}{*}{0.008} \\
\hline 0 & $77(73.3)$ & $32(60.4)$ & \\
\hline 1 & $15(14.3)$ & $10(18.9)$ & \\
\hline 2 & $5(4.8)$ & $10(18.9)$ & \\
\hline 3 & $1(1.0)$ & 0 & \\
\hline $\begin{array}{l}\text { Maintenance of SDAI } \leq 2 \text { with no individual component }>1 \\
\text { and rectal bleeding }=0, n(\%)\end{array}$ & $77(73.3)$ & $27(50.9)$ & 0.009 \\
\hline Cumulative relapse-free probability (SE) & $0.76(0.04)$ & $0.52(0.07)$ & 0.005 \\
\hline
\end{tabular}

ITT intent-to-treat, $M G$ mesalamine granules, SDAI Sutherland Disease Activity Index, SE standard error

${ }^{\text {a }}$ Month $6=$ end of 1 treatment in Phase 3 double-blind treatment phase

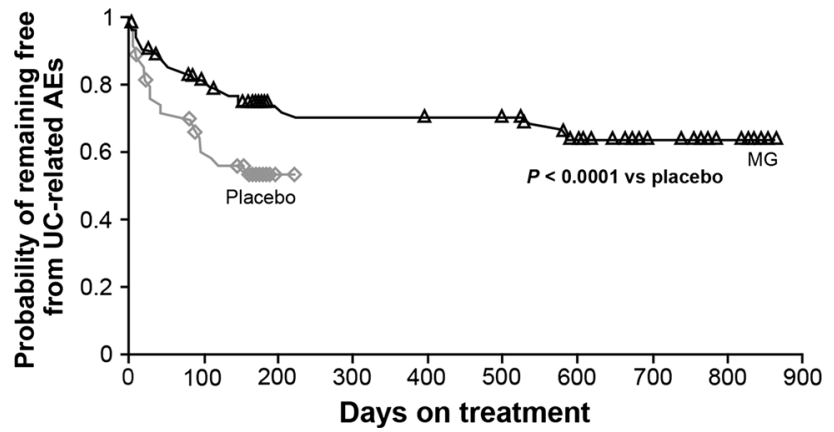

Fig. 3 Probability of remaining free from ulcerative colitis (UC)related adverse events (AEs; defined as UC, hematochezia, or frequent bowel movements) during short-term treatment (MPUC3003 and MPUC3004, pooled) and long-term treatment (MPUC3005). $M G$ mesalamine granules

AEs were relatively uncommon $(<5 \%)$ in patients previously treated with corticosteroids receiving MG $1.5 \mathrm{~g}$ once daily for up to 30 months.

\section{Discussion}

In this pooled analysis, MG administered once daily provided long-term protection against relapse and UC-related AEs for up to 30 months (the duration of the studies) in patients previously induced into remission with corticosteroid therapy for UC flares. These findings are comparable to the results from the overall population of the two double-blind trials (which included the corticosteroid population assessed in the current analysis), wherein the UC relapse-free rate at 6 months was significantly higher in patients treated with MG compared with placebo (79.4 vs. $62.4 \%$, respectively; $P<0.001)[9,10]$. In the current analysis at 6 months, patients previously treated with corticosteroids also experienced a significantly higher relapse-free rate in the MG group (77.1\%) compared with placebo (54.7\%; $P=0.006$ ), supporting $\mathrm{MG}$ as being efficacious in maintaining UC remission in this patient population. 
Table 3 Overall AE summary of patients previously treated with corticosteroids for ulcerative colitis during the double-blind and open-label extension trials

\begin{tabular}{|c|c|c|c|}
\hline \multirow[t]{2}{*}{ Adverse events, $\%$ (rate $^{a}$ ) } & \multicolumn{2}{|c|}{ Short-term safety population $(N=154)$} & \multirow{2}{*}{$\begin{array}{l}\text { Long-term safety population } \\
\text { MG } 1.5 \mathrm{~g} / \text { day }(N=74) \\
\mathrm{PYE}^{\mathrm{b}}=33.4^{\mathrm{c}}\end{array}$} \\
\hline & $\begin{array}{l}\text { MG } 1.5 \mathrm{~g} / \text { day }(n=102) \\
\text { PYE }^{\mathrm{b}}=40.2\end{array}$ & $\begin{array}{l}\text { Placebo }(n=52) \\
\text { PYE }^{b}=16.1\end{array}$ & \\
\hline Any $\mathrm{AE}$ & $65.7(6.0)$ & $65.4(5.8)$ & $81.1(9.6)$ \\
\hline Serious AEs & $2.9(0.1)$ & $1.9(0.1)$ & $10.8(0.3)$ \\
\hline Drug-related AEs & $10.8(0.5)$ & $9.6(0.4)$ & $10.8(0.5)$ \\
\hline AEs leading to discontinuation & $16.7(0.4)$ & $13.5(0.4)$ & $12.2(0.3)$ \\
\hline Deaths & 0 & 0 & 0 \\
\hline \multicolumn{4}{|l|}{$\begin{array}{l}\text { AEs reported in }>5 \% \text { of } \\
\text { MG-treated patients, } \%\left(\text { rate }^{\mathrm{a}}\right)\end{array}$} \\
\hline Headache & $11.8(0.6)$ & $5.8(0.2)$ & $13.5(1.0)$ \\
\hline Diarrhea & $7.8(0.4)$ & $5.8(0.3)$ & $9.5(0.5)$ \\
\hline Upper abdominal pain & $5.9(0.3)$ & 0 & $6.8(0.5)$ \\
\hline Ulcerative colitis & $17.6(0.5)$ & $30.8(1.1)$ & $14.9(0.3)$ \\
\hline Influenza & $5.9(0.2)$ & $1.9(0.1)$ & $9.5(0.3)$ \\
\hline Upper respiratory tract infection & $3.9(0.2)$ & 0 & $8.1(0.3)$ \\
\hline Sinusitis & $2.9(0.1)$ & $1.9(0.2)$ & $8.1(0.2)$ \\
\hline Abdominal pain & $6.9(0.2)$ & $11.5(0.4)$ & $8.1(0.3)$ \\
\hline Constipation & $6.9(0.2)$ & $1.9(0.1)$ & $8.1(0.2)$ \\
\hline Nasopharyngitis & 0 & $3.8(0.1)$ & $8.1(0.2)$ \\
\hline Viral respiratory tract infection & $1.0(0)$ & 0 & $8.1(0.2)$ \\
\hline Nausea & $5.9(0.2)$ & $1.9(0.1)$ & $6.8(0.2)$ \\
\hline Loose stools & $1.0(0)$ & $5.8(0.6)$ & $5.4(0.1)$ \\
\hline Insomnia & $2.9(0.1)$ & 0 & $5.4(0.1)$ \\
\hline
\end{tabular}

$A E$ adverse event, $M G$ mesalamine granules

${ }^{\text {a }}$ Rate $=$ events $\div$ PYE

b PYE $=$ person-years of exposure ([mean exposure in days $\div 365.25] \times$ no. of patients)

${ }^{c}$ Data reflect AEs during time in open-label extension

Although relapse-free rates were not prospectively assessed throughout the course of the long-term OLE trial (e.g., using the SDAI), monitoring of UC-related AEs in patients previously treated with corticosteroids provided further evidence of the short- and long-term benefits of MG. In this pooled analysis, the lower rate of UC-related AEs with MG versus placebo during the 6-month, doubleblind treatment period was sustained in patients continuing to receive $\mathrm{MG}$ once daily for up to 30 months. Thus, consideration of UC-related AE rates over time may offer a long-term surrogate for assessing the potential benefit of MG.

It is noteworthy that, for the 74 MG-treated patients in the long-term safety population and the $102 \mathrm{MG}$-treated patients in the short-term population, the reported incidence of a UC flare AE was similar (14.9 and $17.6 \%$, respectively), with both incidences nearly half the reported incidence observed in the placebo-treated ITT population $(30.8 \%)$. Further examination of UC flare AE rates based on PYE revealed a low rate ( 0.3 events/PYE) after MG treatment for up to 30 months, which was approximately one-third the rate observed in patients receiving placebo (1.1 events/PYE) in the double-blind trials.

This analysis also demonstrated that MG was well tolerated for up to 30 months in patients with UC who had received prior corticosteroid therapy. The percentage of patients treated with MG $1.5 \mathrm{~g}$ once daily with a drugrelated $\mathrm{AE}$ in the long-term safety population was similar to that observed in the short-term safety population (both $10.8 \%$ ), and comparable to findings from the overall study population in the original double-blind trials (10.6\%). Headache was the only non-GI-related AE reported in $>10 \%$ of MG-treated patients, (11.8 and $13.5 \%$ of patients in short- and long-term safety populations, respectively). Hepatic and renal system-related AEs were infrequently observed with long-term MG use. Overall, results were similar to those observed in the overall population of the two double-blind trials [9], as well as other trials of MG for maintenance and remission of UC [10, 15]. Safety results for MG from this study also were consistent with those reported for the OLE (long-term) study, in that 
some AEs occurred early after starting therapy and decreased over time [12].

The role of systemic corticosteroids in the management of UC continues to evolve, although these drugs are typically reserved for inducing remission in patients who are refractory to oral 5-ASAs in combination with topical therapy, or for patients with moderate-to-severe symptoms who require rapid improvement [2]. Despite their benefits, it is widely recognized that prolonged use of systemic corticosteroids may expose patients to potential toxicities and occasionally result in severe complications. These complications may include cushingoid features, metabolic disturbances, emotional and psychiatric disturbances, opportunistic infections, impaired wound healing, striae, glaucoma, cataracts, and osteoporosis [2, 16, 17]. Accordingly, to minimize the risk of steroid-induced AEs in patients with UC, it is recommended that corticosteroid administration be restricted to short-term use only and at the lowest possible effective dose [17].

Another dilemma with corticosteroid use in UC patients is the highly recognized "rebound effect" that often occurs following tapering or discontinuation when switching to alternative therapies [11]. As such, there is a need for agents to be identified that may minimize or prevent steroid-induced UC rebound, prolong the duration of remission, and reduce the need for future systemic corticosteroid therapy, especially for patients with moderate-to-severe disease. The current data from this study support MG administration for the long-term maintenance of UC remission in a population of patients previously treated with corticosteroids to induce remission.

The lack of objective response measurements (SDAI), the lack of availability of placebo-controlled data after 6 months, and the small patient population assessed during the OLE trial (long-term population) were limitations of this study. In addition, conclusions suggesting the role of $\mathrm{MG}$ in steroid switching or tapering cannot be decisively drawn from this post hoc analysis because patients did not immediately switch or taper corticosteroid therapy and begin $\mathrm{MG}$ or placebo therapy in the current study. One eligibility requirement for both double-blind trials was that patients were not permitted to use corticosteroids within 30 days of screening. In addition, as patients were in UC remission at enrollment, initial disease severity scores prior to use of steroid therapy were not available. Thus, disease severity at the time of steroid-treated UC flare (i.e., mild versus moderate) could have theoretically impacted maintenance of remission during treatment. Therefore, the results from this study are considered exploratory and need validation with prospective trials, and the generalizability of these findings should be further explored.
In conclusion, this pooled post hoc analysis demonstrated that MG provides long-term (up to 30 months) maintenance of UC remission in patients with a history of prior corticosteroid therapy for UC flares or maintenance of $\mathrm{UC}$ remission. Accordingly, treatment with once-daily $\mathrm{MG}$ may allow for avoidance of or reduction in corticosteroid use in the maintenance of remission for patients with moderate-to-severe UC. Future large, well-designed, prospective clinical trials are necessary to fully validate these initial observations suggesting that there is long-term benefit with using MG for maintenance of remission in patients with UC who received corticosteroids to induce remission.

Acknowledgments Writing and editorial support were provided under the direction of the authors by Mary Beth Moncrief, Ph.D., and Teresa Tartaglione, Pharm.D., of Synchrony Medical Communications, LLC, West Chester, PA and funded by Salix, a Division of Valeant Pharmaceuticals North America LLC, Bridgewater, NJ, USA.

Funding This study was funded in full by Salix, a Division of Valeant Pharmaceuticals North America LLC, Bridgewater, NJ, USA.

\section{Compliance with ethical standards}

Conflict of interest Gary R. Lichtenstein has served as a consultant for and received research funding from Alaven; Centocor Ortho Biotech; Ferring; Proctor \& Gamble; Prometheus Laboratories, Inc.; Salix, a Division of Valeant Pharmaceuticals North America LLC, Bridgewater, NJ, USA; Shire Pharmaceuticals, UCB and Warner Chilcott. He has also served as a consultant for Abbott Laboratories; Elan; Hospira; Meda Pharmaceuticals; Millennium Pharmaceuticals, Inc.; Pfizer; Santarus, Inc., a former subsidiary of Salix; ScheringPlough; Takeda Pharmaceuticals U.S.A., Inc. and Wyeth, and in a research capacity for Bristol-Myers Squibb. Glenn L. Gordon serves as principal investigator on a variety of research protocols for Salix; serves as a consultant to and on the speakers' bureau for AbbVie, Aptalis, Janssen Pharmaceuticals, Inc., Prometheus, Salix, sanofiaventis U.S., Takeda Pharmaceutical Company Limited, UCB, and Warner Chilcott; and receives research grants from AbbVie, Aptalis, Avaxia, Coronado, Cubist, Dr. Falk, Evoke, Ferring, Furiex, GlaxoSmithKline, Hutchinson, Janssen Pharmaceuticals, Inc., Lexicon, Pfizer Inc., Prometheus, Red Hill BioPharma, Revogenex, Salix, sanofi-aventis U.S., Shire, Takeda Pharmaceutical Company Limited, Theravance, Tranzyme, UBC, UCB, Ventrus, and Warner Chilcott. Salam Zakko serves as a principal investigator on several studies sponsored by Salix. He also is a consultant and speaker for the company, and is a recipient of grants from the company. Additionally, he is a consultant, speaker, and investigator for Novartis, Pfizer, and GlaxoSmithKline. Uma Murthy has no conflicts to disclose. Shahriar Sedghi has no conflicts to disclose. Ronald Pruitt has no conflicts to disclose. Andrew C. Barrett, Enoch Bortey, Craig Paterson, and William P. Forbes are former employees of Salix.

Ethical approval All procedures performed in studies involving human participants were in accordance with the ethical standards of the institutional and/or national research committee and with the 1964 Helsinki declaration and its later amendments or comparable ethical standards.

Open Access This article is distributed under the terms of the Creative Commons Attribution-NonCommercial 4.0 International 
License (http://creativecommons.org/licenses/by-nc/4.0/), which permits any noncommercial use, distribution, and reproduction in any medium, provided you give appropriate credit to the original author(s) and the source, provide a link to the Creative Commons license, and indicate if changes were made.

\section{References}

1. Langan RC, Gotsch PB, Krafczyk MA, Skillinge DD. Ulcerative colitis: diagnosis and treatment. Am Fam Physician. 2007;76:1323-1330.

2. Kornbluth A, Sachar DB, The Practice Parameters Committee of the American College of Gastroenterology. Ulcerative colitis practice guidelines in adults: American College of Gastroenterology, Practice Parameters Committee. Am J Gastroenterol. 2010;105:501-523.

3. Meier J, Sturm A. Current treatment of ulcerative colitis. World J Gastroenterol. 2011;17:3204-3212.

4. Loftus EV Jr. Clinical epidemiology of inflammatory bowel disease: incidence, prevalence, and environmental influences. Gastroenterology. 2004;126:1504-1517.

5. Everhart JE, Ed. The Burden of Digestive Diseases in the United States. Washington, DC: United States Department of Health and Human Services; Public Health Service, National Institutes of Health, National Institute of Diabetes and Digestive and Kidney Diseases; 2008. http://www2.niddk.nih.gov/NR/rdonlyres/0B814 CEA-222A-4677-9B38-89D3BC34E1F4/0/BurdenDD_US_Book marks_Jan2009.pdf. Accessed February 12, 2013. Report no. NIH Publication No. 09-6443.

6. Kappelman MD, Rifas-Shiman SL, Porter CQ, et al. Direct health care costs of Crohn's disease and ulcerative colitis in United States children and adults. Gastroenterology. 2008;135:19071913.

7. Apriso ${ }^{\circledR}$ (mesalamine) extended-release capsules [package insert]. Raleigh, NC: Salix Pharmaceuticals, Inc.; 2012.
8. Lichtenstein GR. Mesalamine in the treatment of ulcerative colitis: novel therapeutic options. Gastroenterol Hepatol. 2009;5:65-73.

9. Lichtenstein GR, Gordon GL, Zakko S, et al. Clinical trial: oncedaily mesalamine granules for maintenance of remission of ulcerative colitis-a 6-month placebo-controlled trial. Aliment Pharmacol Ther. 2010;32:990-999.

10. Lichtenstein GR, Zakko S, Gordon GL, et al. Mesalazine granules $1.5 \mathrm{~g}$ once-daily maintain remission in patients with ulcerative colitis who switch from other 5-ASA formulations: a pooled analysis from two randomised controlled trials. Aliment Pharmacol Ther. 2012;36:126-134.

11. Porro GB, Cassinotti A, Ferrara E, Maconi G, Ardizzone S. Review article: the management of steroid dependency in ulcerative colitis. Aliment Pharmacol Ther. 2007;26:779-794.

12. Lichtenstein GR, Barrett AC, Bortey E, Paterson C, Forbes WP. Long-term safety and tolerability of once-daily mesalamine granules in the maintenance of remission of ulcerative colitis. Inflamm Bowel Dis. 2014;20:1399-1406.

13. Mesalamine pellet to maintain remission of mild to moderate ulcerative colitis: NCT00767728. ClinicalTrials.gov. December 21, 2009. http://www.clinicaltrials.gov/ct2/show/NCT0076772 8?term=NCT00767728\&rank=1. Accessed February 5, 2013.

14. Sutherland LR, Martin F, Greer S, et al. 5-aminosalicylic acid enema in the treatment of distal ulcerative colitis, proctosigmoiditis, and proctitis. Gastroenterology. 1987;92:1894-1898.

15. Moss AC, Peppercorn MA. The risks and the benefits of mesalazine as a treatment for ulcerative colitis. Expert Opin Drug Saf. 2007;6:99-107.

16. De Cassan C, Fiorino G, Danese S. Second-generation corticosteroids for the treatment of Crohn's disease and ulcerative colitis: more effective and less side effects? Dig Dis. 2012;30:368-375.

17. Rutgeerts PJ. Review article: the limitations of corticosteroid therapy in Crohn's disease. Aliment Pharmacol Ther. 2001;15: $1515-1525$. 